\title{
Parameter Estimation of Permanent Magnet Synchronous Motor Using Orthogonal Projection and Recursive Least Squares Combinatorial Algorithm
}

\author{
Iman Yousefi and Mahmood Ghanbari \\ Department of Electrical Engineering, Aliabad Katoul Branch, Islamic Azad University, Aliabad Katoul, Iran \\ Correspondence should be addressed to Mahmood Ghanbari; ghanbari@aliabadiau.ac.ir
}

Received 7 January 2015; Revised 23 June 2015; Accepted 25 June 2015

Academic Editor: Tomasz Kapitaniak

Copyright ( $\odot 2015$ I. Yousefi and M. Ghanbari. This is an open access article distributed under the Creative Commons Attribution License, which permits unrestricted use, distribution, and reproduction in any medium, provided the original work is properly cited.

\begin{abstract}
This paper presents parameter estimation of Permanent Magnet Synchronous Motor (PMSM) using a combinatorial algorithm. Nonlinear fourth-order space state model of PMSM is selected. This model is rewritten to the linear regression form without linearization. Noise is imposed to the system in order to provide a real condition, and then combinatorial Orthogonal Projection Algorithm and Recursive Least Squares (OPA\&RLS) method is applied in the linear regression form to the system. Results of this method are compared to the Orthogonal Projection Algorithm (OPA) and Recursive Least Squares (RLS) methods to validate the feasibility of the proposed method. Simulation results validate the efficacy of the proposed algorithm.
\end{abstract}

\section{Introduction}

Permanent Magnet Synchronous Motor (PMSM) is a good choice to use in robotics industries, electric vehicles, petrochemical industries, sea, and so forth due to large torque to inertia ratio and high power density $[1-5]$ and is a serious opponent to the induction motor $[6,7]$. Regarding widespread application of such motors, design of high speed and high accuracy controllers is of great concern for engineers [8]. The selected model and knowing the parameters of the system are important factors in design of such controllers. The pertaining model has to be accurate enough to define the physical process of the system [4]. PMSM is a nonlinear system. If the linearization in the operating point by choosing improper nonlinear model does not provide appropriate dynamics, the speed and response speed of the controller would be slowed down [9]. Sensitivity of the machine's parameter depends on various factors such as high temperature, mechanical vibrations, loading condition, increase in the service time of the motor, and environmental factors that would cause parametric uncertainty $[3,8,10]$ and has considerable effect on the static and dynamic performance of the system [11]. Robustness to parametric uncertainty has to be imposed by correct estimation of PMSM parameters. Generally, there are two kinds of estimation to find out unknown parameters: online and offline estimation. Offline estimation is carried out when machine is not performing but online estimation is carried out when machine is operating and in steady-state [12]. Online estimation is a very important necessity for such systems. Extended Kalman Filter, ModelReference method, Recursive Least Squares method, neural networks, adaptive algorithms, and decoupling control algorithms are of the online methods to estimate the parameters of PMSM [2, 10, 13-19].

In this paper, we have presented combinatorial estimation methods, that is, Orthogonal Projection Algorithm (OPA) and Recursive Least Squares (RLS). OPA is presented well in [20] to estimate the parameters of PMSM which is very efficient for noise-free systems. The combinatorial OPA\&RLS method would be a good candidate for systems with high noise which are more like real systems. This method can be very helpful for online estimation. In this paper, a nonlinear fourth-order PMSM model is chosen and load angle is selected as accessible output, and voltages $v_{d}, v_{q}$ are selected as input (framed voltages of Park transformation). This nonlinear model is rewritten to linear regression form 
without linearization or simplification. Both estimators can be applied independently but the combinatorial method has the features of both methods. OPA leaps quickly toward the aim parameter with each estimation it does and after that RLS adapts the estimated parameter to the real parameter due to its robustness toward noise.

Section 2 describes state space model transformation of PMSM. In Section 3, the proposed estimator is presented. Simulation results are illustrated in Section 4 and finally the paper is concluded in Section 5.

\section{Transforming the Nonlinear Model to Linear Regression Form}

To achieve high performance for PMSM, the fourth-order state space model of the system is selected under Park transformation $[21,22]$ :

$$
\begin{aligned}
& \dot{x}=A x+B u+f(x), \\
& x=\left[\begin{array}{llll}
x_{1} & x_{2} & x_{3} & x_{4}
\end{array}\right]^{T}=\left[\begin{array}{llll}
\delta & \omega_{e} & i_{q} & i_{d}
\end{array}\right]^{T}, \\
& u=\left[\begin{array}{ll}
u_{1} & u_{2}
\end{array}\right]^{T}=\left[\begin{array}{ll}
v_{q} & v_{d}
\end{array}\right]^{T}, \\
& A=\left[\begin{array}{cccc}
0 & 1 & 0 & 0 \\
0 & 0 & \frac{1.5 p \lambda_{f}}{J} & 0 \\
0 & -\frac{-\lambda_{f} p}{L_{q}} & -\frac{-R}{L_{q}} & 0 \\
0 & 0 & 0 & -\frac{R}{L_{d}}
\end{array}\right] \text {, } \\
& B=\left[\begin{array}{cc}
0 & 0 \\
0 & 0 \\
\frac{1}{L_{q}} & 0 \\
0 & \frac{1}{L_{d}}
\end{array}\right], \\
& f=\left[\begin{array}{c}
0 \\
\frac{1.5 p\left(L_{d}-L_{q}\right) i_{q} i_{d}}{J} \\
-\frac{L_{d} p \omega_{e} i_{d}}{L_{q}} \\
\frac{L_{q} p \omega_{e} i_{q}}{L_{d}}
\end{array}\right] .
\end{aligned}
$$

System inputs are $v_{d}, v_{q}$ Park transformation voltages, $i_{q}, i_{d}$, $\omega_{e}$, and $\delta$ state variables of the system which are rotor angular position, rotor angular speed, and Park transformation currents, respectively.

Physical parameters of the system are $R$ stator resistance, $p$ pole-pairs, $\lambda_{f}$ magnet flux linkage, $J$ inertia coefficient, and $L_{d}, L_{q}$ direct and quadrature inductances of Park transformation, respectively.

The aim of this section is to transform the nonlinear state space model of PMSM to the linear regression form without linearization or simplification techniques. As the linear regression form is expressed in terms of an output, the pertaining output is rewritten in terms of state variables. The selected output is power angle $\left(\delta=x_{1}\right)$.

Equation (3) is obtained by considering $i_{d}, i_{q}$ according to (2) [23] and discretization based on $\left(\dot{x}=\left[x\left(t+T_{s}\right)-x(t)\right] / T_{s}\right)$ in which $T_{s}$ is the sampling time. Consider

$$
\begin{aligned}
i_{d}= & i_{m} \cos (\delta)=i_{m} \cos \left(x_{1}\right), \\
i_{q}= & i_{m} \sin (\delta)=i_{m} \sin \left(x_{1}\right), \\
x_{1}\left[k+T_{s}\right]= & x_{1}[k]+T_{s} x_{2}[k], \\
x_{2}\left[k+T_{s}\right]= & x_{2}[k]+T_{s}\left(\frac{1.5 P \lambda_{f}}{J}\right) x_{3}[k] \\
& +T_{s}\left(\frac{1.5\left(L_{d}-L_{q}\right)}{J}\right) x_{3}[k] x_{4}[k], \\
x_{3}\left[k+T_{s}\right]= & T_{s}\left(\frac{-\lambda{ }_{f} P}{L_{q}}\right) x_{2}[k] \\
& +T_{s}\left(\frac{-R}{L_{q}}\right) i_{m} \sin \left(x_{1}[k]\right)+\frac{T_{s}}{L_{q}} u_{1}[k] \\
& +\frac{T_{s}}{L_{d}} u_{2}\left(P \frac{L_{q}}{L_{d}}\right) i_{m} \sin \left(x_{1}[k]\right) x_{2}[k] . \\
& +T_{s}\left(-p \frac{L_{d}}{L_{q}}\right) i_{m} \cos \left(x_{1}[k]\right) x_{2}[k] \\
& +i_{m} \sin \left(x_{1}[k]\right), \\
x_{4}\left[k+T_{s}\right]= & i_{m} \cos \left(x_{1}[k]\right)+T_{s}\left(\frac{-R}{L_{d}}\right) i_{m} \cos \left(x_{1}[k]\right) \\
& \\
& \\
& \\
& \\
&
\end{aligned}
$$

Rewriting (3) to the linear regression form is needed in a way that $x_{1}$ is written in terms of all state variables. After applying estimator on linear regression form and estimation of the parameters, complicated nonlinear equations have to be solved to obtain physical parameters $\left(R, p, \lambda_{f}, J, L_{q}\right.$, and $\left.L_{d}\right)$. To solve this problem, two linear regression forms are required by a heuristic method and defining new variables in (3) and (2). Therefore, physical parameters can be solved from linear regression without solving nonlinear equation.

2.1. Model Number 1. The second state variable of (3) can be rewritten by using (2) as below:

$$
\begin{aligned}
x_{2}\left[k+T_{s}\right] & \\
= & x_{2}[k]+T_{s}\left(\frac{1.5 P \lambda_{f}}{J}\right) x_{3}[k] \\
& +T_{s}\left(\frac{1.5\left(L_{d}-L_{q}\right)}{J}\right) \frac{i_{m}^{2}}{2} \sin 2\left(x_{1}[k]\right) .
\end{aligned}
$$


By shifting forward in (4) and putting $x_{3}[k+1]$ in it from (3) and substituting $T_{s}=1$,

$$
\begin{aligned}
x_{2}[k & +2] \\
= & x_{2}[k+1] \\
& +\left(\frac{1.5\left(L_{d}-L_{q}\right) i_{m}^{2}}{2 J}\right) \sin 2\left(x_{1}[k+1]\right) \\
& +\left(\frac{1.5 P \lambda_{f}}{J}\right)\left(\frac{-\lambda_{f} P}{L_{q}}\right) x_{2}[k] \\
& +\left(\frac{1.5 P \lambda_{f}}{J}\right)\left(\frac{-R}{L_{q}}\right) \sin \left(x_{1}[k]\right) \\
& +\left(\frac{1.5 P \lambda_{f}}{L_{q} J}\right) u_{1}[k] \\
& +\left(\frac{1.5 P \lambda_{f}}{J}\right)\left(-p \frac{L_{d}}{L_{q}}\right) \cos \left(x_{1}[k]\right) x_{2}[k] \\
& +\left(\frac{1.5 P \lambda_{f} i_{m}}{J}\right) \sin \left(x_{1}[t]\right) .
\end{aligned}
$$

Equation (5) is rewritten in terms of load angle by using the relation between the first and second state variables:

$$
\begin{aligned}
& x_{1}[k+3]-x_{1}[k+2]=x_{1}[k+2]-x_{1}[k+1] \\
& +\left(\frac{1.5 P \lambda_{f}}{J}\right)\left(\frac{-\lambda_{f} P}{L_{q}}\right)\left(x_{1}[k+1]-x_{1}[k]\right) \\
& +\left(\frac{1.5 P \lambda_{f}}{J}\right)\left(\frac{-R}{L_{q}}\right) \sin \left(x_{1}[k]\right)+\left(\frac{1.5 P \lambda_{f}}{L_{q} J}\right) \\
& \cdot u_{1}[k]+\left(\frac{1.5 P \lambda_{f}}{J}\right)\left(-p \frac{L_{d}}{L_{q}}\right) \cos \left(x_{1}[k]\right) \\
& \cdot\left(x_{1}[k+1]-x_{1}[k]\right)+\left(\frac{1.5 P \lambda_{f} i_{m}}{J}\right) \sin \left(x_{1}[t]\right) \\
& +\left(\frac{1.5\left(L_{d}-L_{q}\right) i_{m}^{2}}{2 J}\right) \sin 2\left(x_{1}[k+1]\right) .
\end{aligned}
$$

The above equation is rewritten to the below form by defining new variables:

$$
\begin{aligned}
y_{1}[k] & =A_{1} y_{11}+A_{2} y_{12}+A_{3} y_{13}+A_{4} y_{14}+A_{5} y_{15} \\
A_{1} & =\left(\frac{1.5 P \lambda_{f}}{J}\right)\left(\frac{-\lambda_{f} P}{L_{q}}\right), \\
A_{2} & =\left(\frac{1.5 P \lambda_{f}}{J}\right)\left(\frac{-R}{L_{q}}\right)+\left(\frac{1.5 P \lambda_{f} i_{m}}{J}\right), \\
A_{3} & =\left(\frac{1.5 P \lambda_{f}}{L_{q} J}\right)
\end{aligned}
$$

$$
\begin{aligned}
A_{4} & =\left(\frac{1.5 P \lambda_{f}}{J}\right)\left(-p \frac{L_{d}}{L_{q}}\right), \\
A_{5} & =\left(\frac{1.5\left(L_{d}-L_{q}\right) i_{m}^{2}}{2 J}\right), \\
y_{11} & =\left(x_{1}[k+1]-x_{1}[k]\right), \\
y_{12} & =\sin \left(x_{1}[k]\right), \\
y_{13} & =u_{1}[k], \\
y_{14} & =\cos \left(x_{1}[k]\right)\left(x_{1}[k+1]-x_{1}[k]\right), \\
y_{15} & =\sin 2\left(x_{1}[k+1]\right), \\
y_{1}[k] & =x_{1}[k+3]-2 x_{1}[k+2]+x_{1}[k+1] .
\end{aligned}
$$

Finally a nonlinear model of PMSM is written to common linear regression form considering (7) without any linearization in which $A_{1}, \ldots, A_{7}$ are factors that are obtained from multiplying physical parameters of the system and $y_{11}, \ldots, y_{15}$ are variables that are composed of nonlinear functions, input, and output.

Equation (7) can be written to the linear regression matrix.

In the following equation, $\phi_{1}$ is estimator vector for the first regression form and $\theta_{1}$ parameter vector related to this form:

$$
\begin{aligned}
y_{1}[k] & =\phi_{1}^{T} \theta_{1}, \\
\phi_{1} & =\left[\begin{array}{lllll}
y_{11} & y_{12} & y_{13} & y_{14} & y_{15}
\end{array}\right]^{T}, \\
\theta_{1} & =\left[\begin{array}{lllll}
A_{1} & A_{2} & A_{3} & A_{4} & A_{5}
\end{array}\right] .
\end{aligned}
$$

2.2. Model Number 2. The second state variable of (3) can be rewritten by using (2) as below:

$$
\begin{aligned}
x_{2}\left[k+T_{s}\right] & \\
= & x_{2}[k]+T_{s}\left(\frac{1.5 P \lambda_{f} i_{m}}{J}\right) \sin \left(x_{1}[t]\right) \\
& +T_{s}\left(\frac{1.5\left(L_{d}-L_{q}\right) i_{m}}{J}\right) \sin \left(x_{1}[k]\right) x_{4}[t] .
\end{aligned}
$$

By shifting forward in (11) and putting $x_{3}[k+1]$ in it from (3) and substituting $T_{s}=1$,

$$
\begin{aligned}
x_{2}[k+2]= & x_{2}[k+1]+\left(\frac{1.5 P \lambda_{f} i_{m}}{J}\right) \sin \left(x_{1}[k+1]\right) \\
& +\left(\frac{1.5\left(L_{d}-L_{q}\right) i_{m}^{2}}{J}\right) \sin \left(x_{1}[k+1]\right) \\
& \cdot \cos \left(x_{1}[t]\right)+\left(\frac{1.5\left(L_{d}-L_{q}\right) i_{m}}{L_{d} J}\right) u_{2}
\end{aligned}
$$




$$
\begin{aligned}
& +\left(\frac{1.5\left(L_{d}-L_{q}\right) i_{m}^{2}}{J}\right)\left(\frac{-R}{L_{d}}\right) \\
& \cdot \sin \left(x_{1}[k+1]\right) \cos \left(x_{1}[t]\right) \\
& +\left(\frac{1.5\left(L_{d}-L_{q}\right) i_{m}^{2}}{J}\right)\left(P \frac{L_{q}}{L_{d}}\right) \\
& \cdot \sin \left(x_{1}[k+1]\right) \sin \left(x_{1}[k]\right) x_{2}[k] .
\end{aligned}
$$

Equation (12) is rewritten in terms of load angle by using the relation between the first and second state variables:

$$
\begin{aligned}
& x_{1}[k+3]-x_{1}[k+2]=x_{1}[k+2]-x_{1}[k+1] \\
& +\left(\frac{1.5 P \lambda_{f} i_{m}}{J}\right) \sin \left(x_{1}[k+1]\right) \\
& +\left(\frac{1.5\left(L_{d}-L_{q}\right) i_{m}^{2}}{J}\right) \sin \left(x_{1}[k+1]\right) \cos \left(x_{1}[k]\right) \\
& +\left(\frac{1.5\left(L_{d}-L_{q}\right) i_{m}}{L_{d} J}\right) \sin \left(x_{1}[k+1]\right) u_{2} \\
& +\left(\frac{1.5\left(L_{d}-L_{q}\right) i_{m}^{2}}{J}\right)\left(\frac{-R}{L_{d}}\right) \sin \left(x_{1}[k+1]\right) \\
& \cdot \cos \left(x_{1}[k]\right)+\left(\frac{1.5\left(L_{d}-L_{q}\right) i_{m}^{2}}{J}\right)\left(P \frac{L_{q}}{L_{d}}\right) \\
& \cdot \sin \left(x_{1}[k+1]\right) \sin \left(x_{1}[k]\right)\left(x_{1}[k+1]-x_{1}[k]\right) .
\end{aligned}
$$

The above equation is rewritten to the below form by defining new variables:

$$
\begin{aligned}
& y_{2}[k]=B_{1} y_{21}+B_{2} y_{22}+B_{3} y_{23}+B_{4} y_{24}, \\
& B_{1}=\left(\frac{1.5 P \lambda_{f} i_{m}}{J}\right), \\
& B_{2}=\left(\frac{1.5\left(L_{d}-L_{q}\right) i_{m}^{2}}{J}\right)\left(1+\left(\frac{-R}{L_{d}}\right)\right), \\
& B_{3}=\left(\frac{1.5\left(L_{d}-L_{q}\right) i_{m}}{L_{d} J}\right), \\
& B_{4}=\left(\frac{1.5\left(L_{d}-L_{q}\right) i_{m}^{2}}{J}\right)\left(P \frac{L_{q}}{L_{d}}\right), \\
& y_{2}[k]=x_{1}[k+3]-2 x_{1}[k+2]+x_{1}[k+1], \\
& y_{21}=\sin \left(x_{1}[k+1]\right), \\
& y_{22}=\sin \left(x_{1}[k+1]\right) \cos \left(x_{1}[k]\right),
\end{aligned}
$$

$$
\begin{aligned}
& y_{23}=\sin \left(x_{1}[k+1]\right) u_{2}[k], \\
& y_{24} \\
& \quad=\sin \left(x_{1}[k+1]\right) \sin \left(x_{1}[k]\right)\left(x_{1}[k+1]-x_{1}[k]\right) .
\end{aligned}
$$

Finally a nonlinear model of PMSM is written to common linear regression form like form number 1 considering equation (14) without any linearization in which $B_{1}, \ldots, B_{7}$ are factors that are embedded in the physical parameters of the system and $y_{21}, \ldots, y_{24}$ are variables that are composed of nonlinear functions, input, and output.

Equation (14) can be written to the linear regression matrix like form number 1 :

$$
\begin{aligned}
y_{2}[k] & =\phi_{2}^{T} \theta_{2}, \\
\phi_{2} & =\left[\begin{array}{llll}
y_{21} & y_{22} & y_{23} & y_{24}
\end{array}\right]^{T}, \\
\theta_{2} & =\left[\begin{array}{llll}
B_{1} & B_{2} & B_{3} & B_{4}
\end{array}\right] .
\end{aligned}
$$

In (13), $\phi_{2}$ is estimator vector and $\theta_{2}$ parameter vector related to form number 2 .

\section{OPA and RLS Estimator}

RLS is a powerful tool in system identification which is robust against noise, while OPA is an interesting method with high convergence speed with poor performance in noisy environment $[24,25]$. Both advantages of these two methods can be used simultaneously if they are combined. Reference [26] is a good reference to understand the efficacy of the combinatorial method which was applied on synchronous generator and good results were obtained. The aim of this paper is to estimate the physical parameter of the PMSM using OPA\&RLS method in noisy environment with arbitrary initial values of physical parameters. It is noted that nonlinear PMSM system is extremely sensitive to physical parameters variations; hence estimation of parameters of such systems is very important by this algorithm.

Estimation process is started with orthogonal projection and orthogonal base vectors of estimator that are in the same dimension with the number of parameters (subspace of OPA estimation method) which are produced [20, 24, 25]. After producing estimation subspace, RLS is used and last estimation of parameters in OPA method is applied to it which is a good initial estimation for RLS to increase the convergence speed. Then, RLS continues the estimation process with a good accuracy to achieve physical parameters. Stages of the combinatorial algorithm are given in the following:

Stage zero: OPA is started with $t=0$ and $\varepsilon=0$ and selection of initial parameters $\theta_{0}$ and $P_{0}$ covariance identity matrix.

Stage one: a step is added to $t(t=t+1)$ and estimator vector $\phi_{t-1}$ is calculated.

Stage two: if the estimator vector of this stage is not linearly dependent on the estimator vector of the 
TABLE 1: Parameters of the understudy system [27].

\begin{tabular}{lccccc}
\hline$R$ & $L_{q}$ & $L_{d}$ & $\lambda_{f}$ & $j$ & $p$ \\
\hline $2.87 \Omega$ & $9 \mathrm{mH}$ & $7 \mathrm{mH}$ & $0.175 \mathrm{mWb}$ & $8 \mathrm{gm}^{2}$ & 4 \\
\hline
\end{tabular}

previous stage $\left(\phi_{t-1}^{T} p_{t-1} \phi_{t-1} \neq 0\right)$, we return to stage three; otherwise previous values of parameters are substituted $\left(p_{t}=p_{t-1}, \widehat{\theta}_{t}=\widehat{\theta}_{t-1}\right)$ and we go to stage one.

Stage three: $\theta_{t}$ and $p_{t}$ are obtained after recursive equation of (18).

Stage four: if the rank of estimator matrix is equal to the number of parameters $(\operatorname{Rank}(\Phi)=\operatorname{dim}(\theta))$, then OPA is finished in $t_{\mathrm{OPA}}$ and we go to stage five; otherwise we return to stage one.

Stage five: to start the estimation with RLS method, $\varepsilon=1$ and $\theta_{t_{\mathrm{OPA}}}$ are considered as initial estimation.

Stage six: a step is added to $t(t=t+1)$ and estimator vector $\phi_{t-1}$ is calculated.

Stage seven: $\theta_{t}$ and $p_{t}$ are updated using (18).

Stage eight: if the stop condition is not met, we return to stage six. Consider the following:

$$
\begin{aligned}
& \widehat{\theta}_{t}= \widehat{\theta}_{t-1}+\frac{P_{t-1} \cdot \phi_{t-1}}{\varepsilon+\phi_{t-1}^{T} \cdot P_{t-1} \cdot \phi_{t-1}} \\
& \cdot\left[y[t]-\widehat{\theta}_{t-1}^{T} \cdot \phi_{t-1}\right], \\
& P_{t}=P_{t-1}+\frac{P_{t-1} \cdot \phi_{t-1} \cdot \phi_{t-1}^{T} \cdot P_{t-1}}{\varepsilon+\phi_{t-1}^{T} \cdot P_{t-1} \cdot \phi_{t-1}} .
\end{aligned}
$$

\section{Simulation}

To validate the efficiency and accuracy of the combinatorial OPA\&RLS method, the proposed method is implemented on a PMSM with specifications of Table 1 [27].

The signal (PRBS) is applied to the input of system for data production to identify and excite the dynamics and a noise with SNR = 15 is added to simulate a real condition for output of the system (load angle) which is shown in Figure 2 (amplitude and output). Simulation is performed in MATLAB software.

To show the superiority of the proposed method, it is compared to OPA and RLS estimator. The criteria for evaluation are the speed and accuracy estimator in converging to the real parameters in a way that the second norm of parameter estimation error vector $\left(L_{2}\right)$ is minimized:

$$
L_{2}=\|\theta-\hat{\theta}\|_{2} .
$$

These criteria are evaluated and compared for the three methods, that is, OPA, RLS, and OPA\&RLS methods, for both linear regression forms from the second part with the same initial estimation and stop condition.

This criterion is shown in Figure 1 for RLS and OPA\&RLS methods. It is shown that this criterion is first decreased

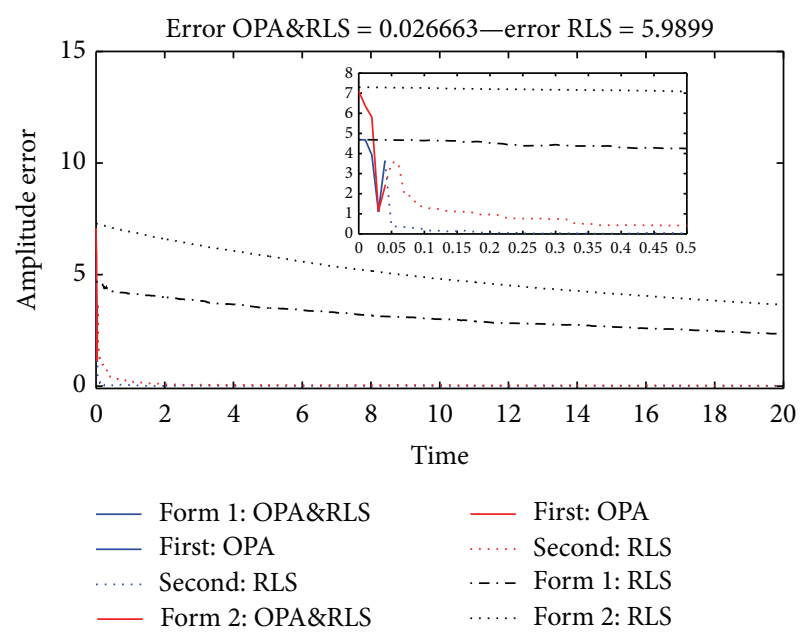

FIGURE 1: Second norm of parameters estimation error in OPA.
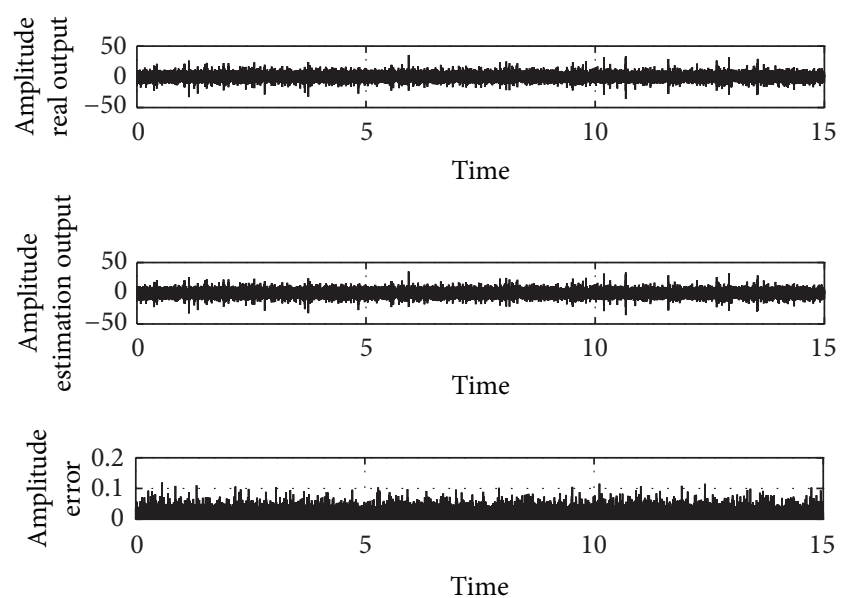

FIGURE 2: The figure shows output of real condition and estimated model and their difference. As shown, estimated and real output.

for OPA method with respect to speed which shows quick convergence toward objective parameters but after that this trend is not preserved and system would oscillate and diverges due to noise. It is shown from Figure 1 that the second norm of factors estimation error for RLS is descending but its slope is slight (low) which requires too many samples for parameters convergence. However, it is prominent that, in OPA\&RLS method, parameters would leap greatly toward the objective parameters with a quick decrease in initial samples and after that it has a proper trend until the convergence to real parameters and appropriate estimation which is very proper for noisy condition. This is because both features of RLS and OPA are used in a single algorithm.

As discussed in Section 2, to avoid nonlinear complicated calculation for estimating physical parameters, two regression forms are defined according to (9) and (13). Now, the values of physical parameters are calculated with simple mathematics and given in Table 2. 
TABLE 2: Comparison between the convergence of OPA and RLS and OPA\&RLS methods with the same condition.

\begin{tabular}{lccccccc}
\hline & $R$ & $L_{q}$ & $L_{d}$ & $\lambda_{f}$ & $j$ & $p$ & Time \\
\hline Real & 2.875 & 9 & 7 & 0.175 & 8 & 4 & - \\
OPA & -0.264 & -0.331 & 8.1717 & 0.1547 & -0.495 & -0.180 & 20 \\
RLS & 1.4925 & 7.1954 & 7.7590 & 0.1911 & 7.8661 & 3.2461 & 20 \\
OPA\&RLS & 2.897 & 9.0650 & 6.9981 & 0.1744 & 8.006 & 4.012 & 20 \\
\hline
\end{tabular}

TABLE 3: Investigation of normalized residue in three algorithms.

\begin{tabular}{lc}
\hline & The normalized residual \\
\hline OPA method & $12.023(\mathrm{~dB})$ \\
RLS method & $3.023(\mathrm{~dB})$ \\
OPA\&RLS method & $-37.56(\mathrm{~dB})$ \\
\hline
\end{tabular}

Figure 2 shows output of real condition and estimated model and their difference. As shown, estimated and real output are alike with agreeable accuracy.

One of the criteria for evaluating the error of estimation is the normalized residue criterion according to $(20)[28,29]$. Here, this criterion is calculated and is given in Table 3. It is observed that the proposed method is superior with respect to other methods. Consider the following:

$$
J_{\mathrm{dB}}=10 \log \left(\frac{\sum_{1}^{N}(y(t)-\hat{y}(t))^{2}}{\sum_{1}^{N} y(t)^{2}}\right)
$$

\section{Conclusion}

In this paper a new combinatorial method of Orthogonal Projection Algorithm and Recursive Least Squares (OPA\&RLS) for parameter estimation of PMSM in noise-covered environment is proposed. To avoid solving complicated nonlinear equation, two linear regression forms of fourth-order state space PMSM were rewritten and OPA\&RLS estimator was compared to OPA and RLS estimators. Speed and accuracy of convergence were investigated in three algorithms and it was shown that OPA\&RLS method has better results with respect to the two single methods. The proposed algorithm can be applied to any linear or nonlinear system where state space model of the system is rewritten to linear regression form. It is also verified that this method is very appropriate for online estimation.

\section{Nomenclature}

\section{Constant}

$R$ : Stator resistance

$p$ : Pole-pairs

$\lambda_{f}$ : Magnet flux linkage

$J:$ Inertia coefficient

$L_{d}$ : Direct and quadrature inductances of Park transformation
$L_{q}:$ Direct and quadrature inductances of Park transformation

$\theta_{1}:$ Parameter vector form 1

$\theta_{2}$ : Parameter vector form 2 .

Variables

$i_{q}:$ Park transformation currents

$i_{d}$ : Park transformation currents

$\omega_{e}$ : Rotor angular speed

$\delta$ : Rotor angular position

$i_{m}$ : Nominal current

$\phi_{1}$ : Estimator vector form 1

$\phi_{2}$ : Estimator vector form 2

$y_{1}$ : Output form 1

$y_{2}$ : Output form 2

$p$ : Covariance matrix

$L_{2}$ : Second norm of parameter estimation error vector

$J$ : Normalized residue.

Indices

$k$ : Step sample time in discrete time

$t$ : Time (sec).

\section{Conflict of Interests}

The authors declare that there is no conflict of interests regarding the publication of this paper.

\section{References}

[1] Y. Xu, U. Vollmer, A. Ebrahimi, and N. Parspour, "Online estimation of the stator resistances of a PMSM with consideration of magnetic saturation," in Proceedings of the International Conference and Exposition on Electrical and Power Engineering (EPE '12), pp. 360-365, IEEE, Iaşi, Romania, October 2012.

[2] K. Liu, Q. Zhang, J. Chen, Z. Q. Zhu, and J. Zhang, "Online multiparameter estimation of nonsalient-pole PM synchronous machines with temperature variation tracking," IEEE Transactions on Industrial Electronics, vol. 58, no. 5, pp. 1776-1788, 2011.

[3] Q. Meng, T. Zhang, J.-F. He, J.-Y. Song, and J.-W. Han, “Dynamic modeling of a 6-degree-of-freedom Stewart platform driven by a permanent magnet synchronous motor," Journal of Zhejiang University: Science C, vol. 11, no. 10, pp. 751-761, 2010.

[4] E. G. Shehata, "Speed sensorless torque control of an IPMSM drive with online stator resistance estimation using reduced order EKF,' International Journal of Electrical Power \& Energy Systems, vol. 47, no. 1, pp. 378-386, 2013.

[5] N. Öztürk and E. Çelik, "Speed control of permanent magnet synchronous motors using fuzzy controller based on genetic algorithms," International Journal of Electrical Power \& Energy Systems, vol. 43, no. 1, pp. 889-898, 2012.

[6] P. Pillay and R. Krishnan, "Modeling of permanent magnet motor drives," IEEE Transactions on Industrial Electronics, vol. 35, no. 4, pp. 537-541, 1988.

[7] O. Aydogmus and S. Sünter, "Implementation of EKF based sensorless drive system using vector controlled PMSM fed by a matrix converter," International Journal of Electrical Power and Energy Systems, vol. 43, no. 1, pp. 736-743, 2012. 
[8] L. Liu and D. A. Cartes, "Synchronisation based adaptive parameter identification for permanent magnet synchronous motors," IET Control Theory and Applications, vol. 1, no. 4, pp. 1015-1022, 2007.

[9] C.-H. Lin and C.-P. Lin, "The hybrid RFNN control for a PMSM drive electric scooter using rotor flux estimator," Electrical Power and Energy Systems, vol. 51, pp. 889-898, 2012.

[10] F. Aymen, N. Martin, L. Sbita, and J. Novak, "Online PMSM parameters estimation for 32000rpm," in Proceedings of the International Conference on Control, Engineering \& Information Technology (CEIT '13), vol. 3, pp. 148-152, Sousse, Tunisia, June 2013.

[11] G. Junli and Z. Yingfan, "Research on parameter identification and PI self-tuning of PMSM," in Proceedings of the 2nd International Conference on Information Science and Engineering (ICISE '10), pp. 5251-5254, December 2010.

[12] G. Gatto, I. Marongiu, and A. Serpi, "Discrete-time parameter identification of a surface-mounted permanent magnet synchronous machine," IEEE Transactions on Industrial Electronics, vol. 60, no. 11, pp. 4869-4880, 2013.

[13] K. Liu and Z. Q. Zhu, "Online estimation of the rotor flux linkage and voltage-source inverter nonlinearity in permanent magnet synchronous machine drives," IEEE Transactions on Power Electronics, vol. 29, no. 1, pp. 418-427, 2014.

[14] Y. Shi, K. Sun, L. Huang, and Y. Li, "Online identification of permanent magnet flux based on extended Kalman filter for IPMSM drive with position sensorless control," IEEE Transactions on Industrial Electronics, vol. 59, no. 11, pp. 4169-4178, 2012.

[15] B. Nahid Mobarakeh, F. Meibody-Tabar, and F. M. Sargos, "Online identification of PMSM electrical parameters based on decoupling control," in Proceedings of the Conference Record of the IEEE Industry Applications Conference 36th IAS Annual Meeting, vol. 1, pp. 266-273, Chicago, Ill, USA, September 2001.

[16] T. Boileau, N. Leboeuf, B. Nahid-Mobarakeh, and F. MeibodyTabar, "Online identification of PMSM parameters: parameter identifiability and estimator comparative study," IEEE Transactions on Industry Applications, vol. 47, no. 4, pp. 1944-1957, 2011.

[17] T. Boileau, B. Nahid-Mobarakeh, and F. Meibody-Tabar, "Online identification of PMSM parameters: model-reference $v s$ EKF"' in Proceedings of the IEEE Industry Applications Society Annual Meeting (IAS '08), pp. 1-8, can, October 2008.

[18] K. Liu, Z. Q. Zhu, and D. A. Stone, "Parameter estimation for condition monitoring of PMSM stator winding and rotor permanent magnets," IEEE Transactions on Industrial Electronics, vol. 60, no. 12, pp. 5902-5913, 2013.

[19] C. Cai, F. Chu, Z. Wang, and K. Jia, "Identification and control of PMSM using adaptive BP-PID neural network," in Advances in Neural Networks, vol. 7952 of Lecture Notes in Computer Science, pp. 155-162, Springer, 2013.

[20] M. Ghanbari, I. Yousefi, and V. Mossadegh, "Online parameter estimation of permanent magnet synchronous motor using orthogonal projection algorithm," Indian Journal Scientific Research, vol. 2, no. 1, pp. 32-36, 2014.

[21] A. K. Parvathy, R. Devanathan, and V. Kamaraj, "Application of quadratic linearization to control of permanent magnet synchronous motor," in Proceedings of the Joint International Conference on Power System Technology and IEEE Power India Conference, pp. 158-163, 2008.

[22] M. A. Hamida, J. de Leon, A. Glumineau, and R. Boisliveau, "An adaptive interconnected observer for sensorless control of PM synchronous motors with online parameter identification,"
IEEE Transactions on Industrial Electronics, vol. 60, no. 2, pp. 739-748, 2013.

[23] R. Krishnan, Permanent Magnet Synchronous and Brushless DC Motor Drives, Taylor \& Francis, 2010.

[24] J. Zhao and I. Kanellakopoulos, "Active identification for discrete-time nonlinear control. I. Output-feedback systems," IEEE Transactions on Automatic Control, vol. 47, no. 2, pp. 210224, 2002.

[25] H. Agahi, M. Karrari, W. Rosehart, and O. P. Malik, "Application of active identification method to synchronous generator parameter estimation," in Proceedings of the IEEE Power Engineering Society General Meeting (PES '06), Montreal, Canada, June 2006.

[26] H. Agahi, Active identification for nonlinear multivariable system identification and its application for synchronous generator identification [Ph.D. thesis], Amirkabir University of Technology, Tehran, Iran, 2007.

[27] J. Chiasson, Modeling and High Performance Control of Electric Machines, IEEE Press Series on Power Engineering, IEEE Press, 2005.

[28] M. Ghanbari and M. Haeri, "Parametric identification of fractional-order systems using a fractional Legendre basis," Proceedings of the Institution of Mechanical Engineers. Part I: Journal of Systems and Control Engineering, vol. 224, no. 3, pp. 261-274, 2010.

[29] M. Ghanbari and M. Haeri, "Order and pole locator estimation in fractional order systems using bode diagram," Signal Processing, vol. 91, no. 2, pp. 191-202, 2011. 


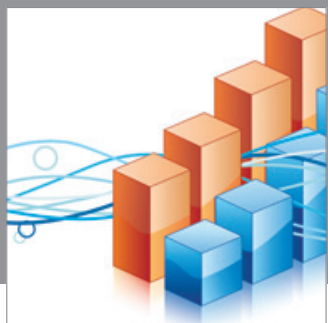

Advances in

Operations Research

mansans

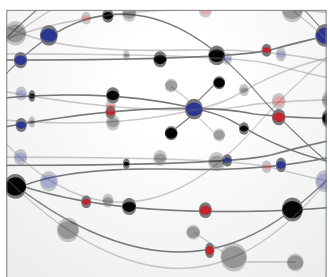

The Scientific World Journal
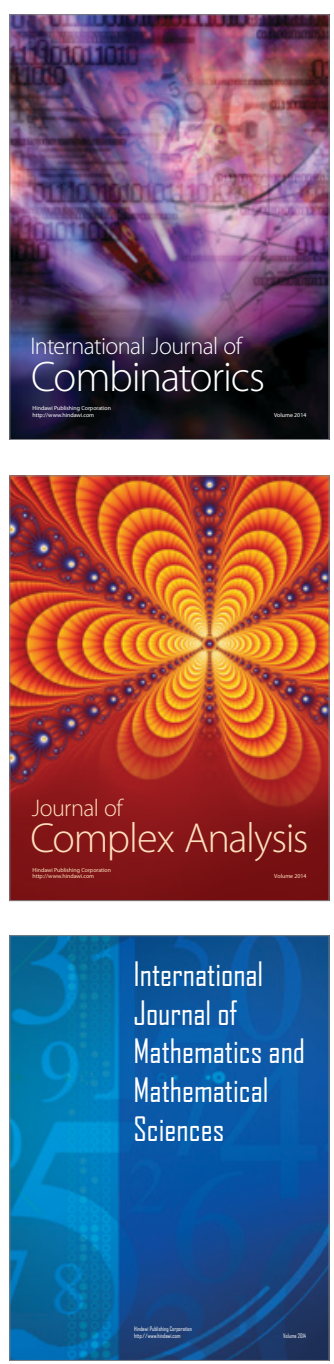
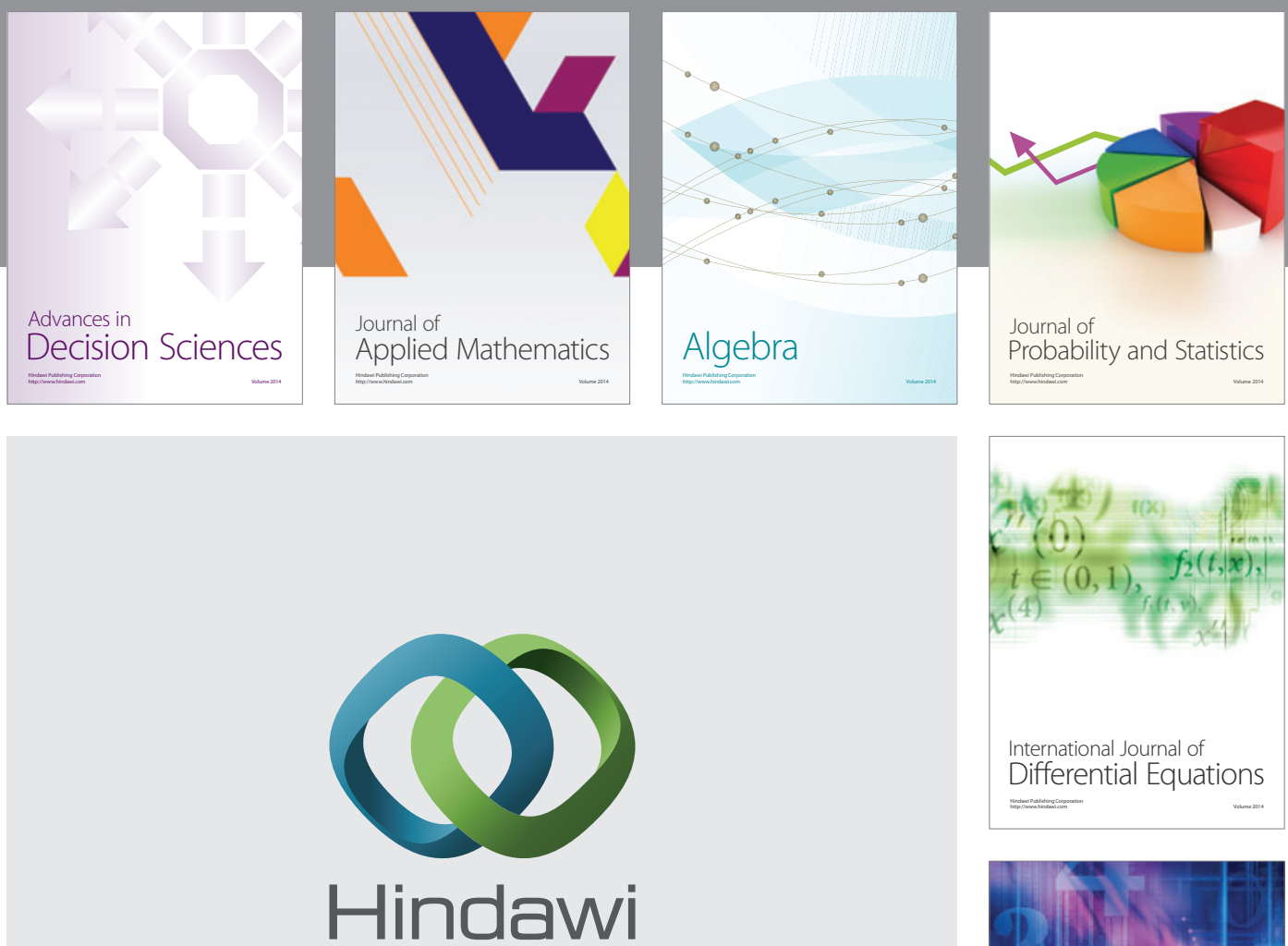

Submit your manuscripts at http://www.hindawi.com
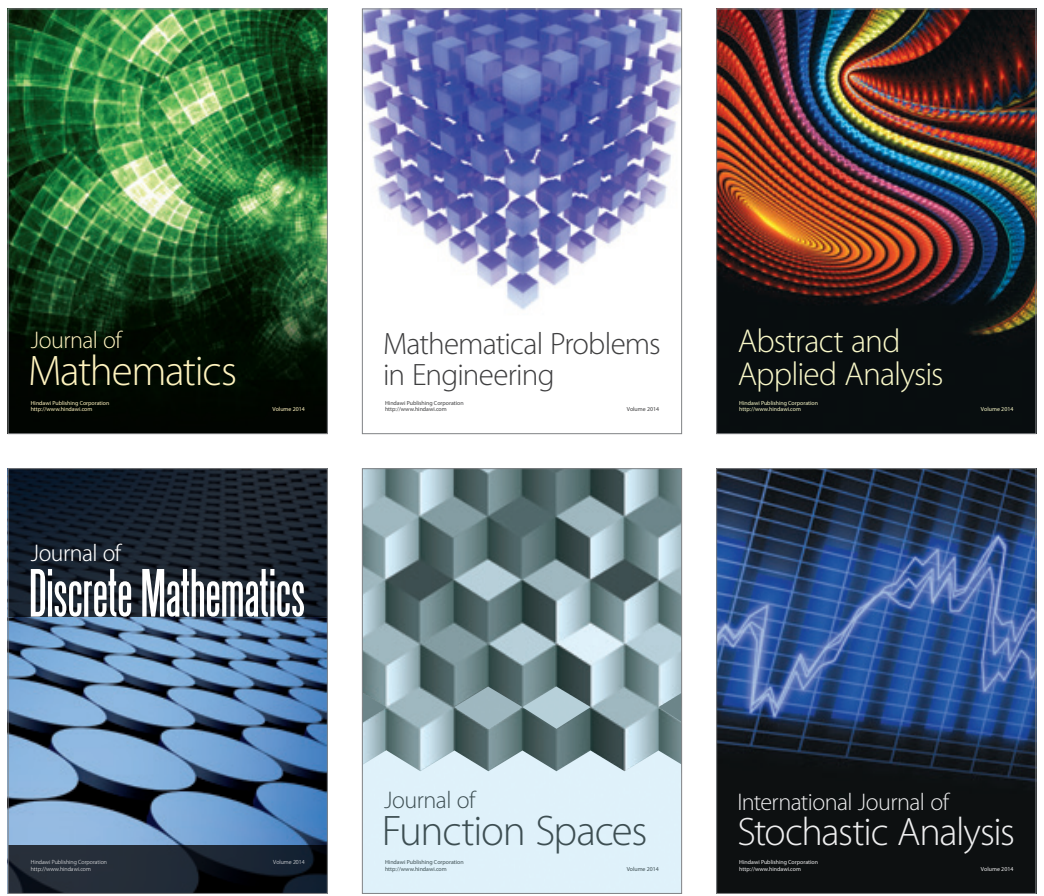

Journal of

Function Spaces

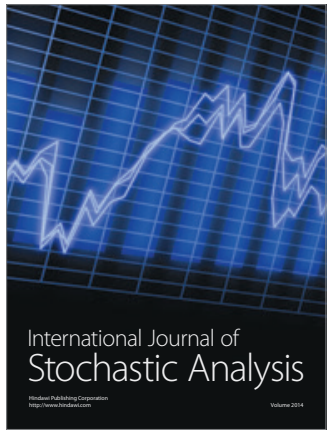

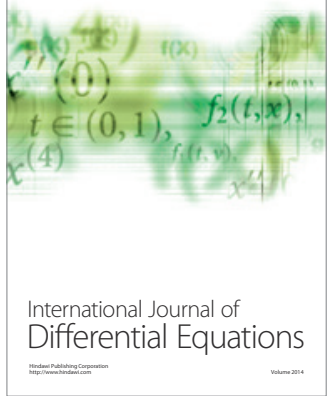
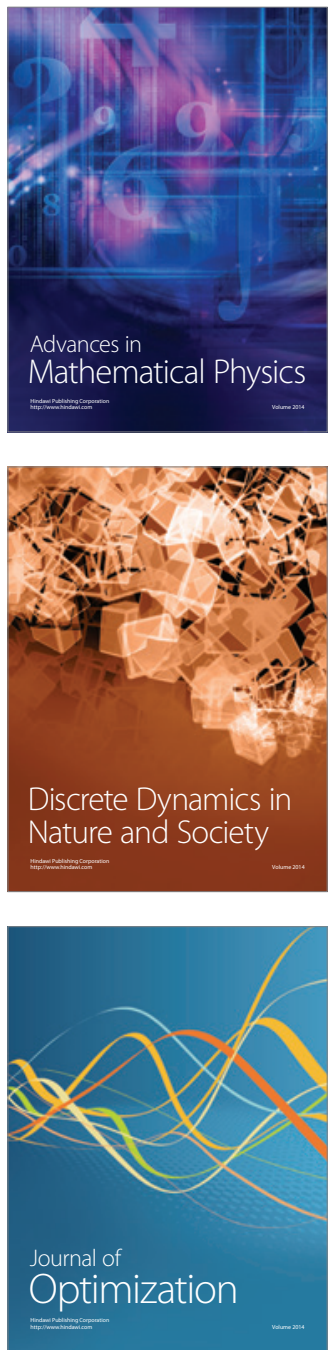